04

\title{
Фотоиндуцированное поглощение и импульсная запись динамических голограмм в кристаллах силиката висмута
}

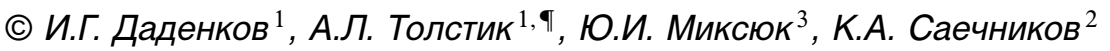 \\ ${ }^{1}$ Белорусский государственный университет, \\ 220030 Минск, Беларусь \\ ${ }^{2}$ Белорусский государственный педагогический университет, \\ 220030 Минск, Беларусь \\ ؟e-mail: Tolstik@bsu.by \\ Поступила в редакцию 15.03.2020 г. \\ В окончательной редакции 15.03.2020 г. \\ Принята к публикации 20.05.2020 г.
}

Исследована динамика фотоиндуцированного поглощения и записи голографических решеток в фоторефрактивных кристаллах силиката висмута. Показано, что при использовании наносекундных лазерных импульсов и интенсивности порядка $1 \mathrm{MW} / \mathrm{cm}^{2}$ и более имеет место наведенное поглощение, обусловленное заселением короткоживущих ловушечных уровней с характерными временами релаксации на уровне единиц и десятков миллисекунд. В этих условиях реализована запись динамических голограмм в кристаллах силиката висмута. Установлены два механизма записи голографических решеток с временами жизни, отличающимися на три порядка. При сравнительно небольших интенсивностях, меньших либо порядка $1 \mathrm{MW} / \mathrm{cm}^{2}$, отклик среды определяется фоторефрактивным механизмом нелинейности с временем релаксации на уровне нескольких секунд. При интенсивностях, превышающих $5 \mathrm{MW} / \mathrm{cm}^{2}$, появляется быстрая компонента (время релаксации миллисекунды), которую можно связать с заселением короткоживущих ловушек. Показано, что вклад каждого механизма существенно зависит от интенсивности лазерного излучения, и при интенсивностях, превышающих $10-15 \mathrm{MW} / \mathrm{cm}^{2}$, определяющую роль играют короткоживущие ловушки с временем жизни порядка миллисекунд.

Ключевые слова: голография, динамические голограммы, фотоиндуцированное поглощение, силлениты, силикат висмута.

DOI: $10.21883 /$ OS.2020.09.49867.90-20

\section{Введение}

Одним из существенных преимуществ кубических фоторефрактивных кристаллов семейства силленитов $\left(\mathrm{Bi}_{12} \mathrm{SiO}_{20}, \mathrm{Bi}_{12} \mathrm{TiO}_{20}, \mathrm{Bi}_{12} \mathrm{GeO}_{20}\right)$ является формирование в них динамических голограмм в реальном времени. Это определяет их использование в адаптивных интерферометрах, системах ассоциативной памяти и усиления оптических изображений, голографических системах записи, хранения и обработки информации и др. [16]. Физические процессы записи голограмм в фоторефрактивных кристаллах основаны на пространственном перераспределении зарядов в поле интерферирующих световых пучков по многочисленным центрам, имеющим разнообразную природу возникновения и характеристики.

По своей энергетической структуре фоторефрактивные кристаллы относятся к широкозонным диэлектрикам, при этом существенное влияние оказывают примеси и структурные дефекты кристаллической решетки, приводящие к возникновению в запрещенной зоне донорных и акцепторных энергетических уровней $[5,7]$. Особенностью является одновременное существование как долгоживущих (секунды, часы), так и короткоживущих ловушек (микро- и миллисекунды) [8-13]. Пе- реходы с уровня, лежащего в запрещенной зоне, в зону проводимости приводят к образованию подвижных носителей заряда, причем такие переходы возможны при использовании излучения с заметно большей длиной волны, чем для прямых межзонных переходов, позволяя работать с излучением в видимой и ближней ИК областях спектра $[5,14]$. Последующая диффузия или дрейф электронов во внешнем электрическом поле обусловливают заселение ловушечных уровней, формирование поля пространственного заряда и модуляцию показателя преломления вследствие эффекта Поккельса. Таким образом формируется голографическая решетка, динамика которой определяется динамикой поля пространственного заряда. Как правило, для формирования динамических голограмм в фоторефрактивных кристаллах используют непрерывное лазерное излучение [1-4,6,7]. Новые возможности открываются при использовании лазерных импульсов нано- и пикосекундной длительности [15-18].

В настоящей работе приведены результаты исследования процессов фотоиндуцированного поглощения и формирования коротко- и долгоживущих голографических решеток в фоторефрактивных кристаллах силиката висмута (BSO) со сложной структурой дефектных центров в условиях импульсного лазерного возбуждения. 


\section{Фотоиндуцированное поглощение}

Фотоиндуцированное поглощение в кристаллах семейства силленитов в видимой и ближней ИК областях спектра определяется заселением ловушечных уровней, лежащих в запрещенной зоне кристалла. Наличие таких уровней актуально для силленитов вследствие присутствия в кристаллах плохо контролируемых примесей и дефектов, существенно влияющих на положение и вероятности заселения коротко- и долгоживущих ловушек. Исследование динамики наведенного поглощения позволяет проанализировать процессы, происходящие в кристаллах при импульсном лазерном воздействии. На рис. 1 представлена типичная осциллограмма прошедшего через кристалл силиката висмута излучения на длине волны $632.8 \mathrm{~nm}$ (гелий-неоновый лазер) после воздействия лазерного импульса длительностью $20 \mathrm{~ns}$ на длине волны $532 \mathrm{~nm}$. Видно существенное уменьшение прошедшей интенсивности на временах порядка сотен миллисекунд, причем можно выделить этап быстрого ( $10 \mathrm{~ms})$ уменьшения наведенного поглощения и этап медленной релаксации (> $100 \mathrm{~ms})$.

Для описания процессов, происходящих в кристалле силиката висмута при импульсном лазерном возбуждении, воспользуемся схемой энергетических состояний и процессов фотовозбуждения и релаксации, представленной на рис. 2 [19]. Наведенное поглощение определяется переходами электронов из валентной зоны 1 в зону проводимости 4 с последующей рекомбинацией на короткоживущие 3 и долгоживущие 2 энергетические уровни (ловушки), расположенные в запрещенной зоне. Ширина запрещенной зоны для кристалла титаната висмута составляет $3.08 \mathrm{eV}$, что не позволяет осуществить прямой межзонный переход при поглощении фотонов на длине волны $532 \mathrm{~nm}$ (энергия кванта $2.33 \mathrm{eV}$ ), используемой в эксперименте. Для объяснения поглощения мощных

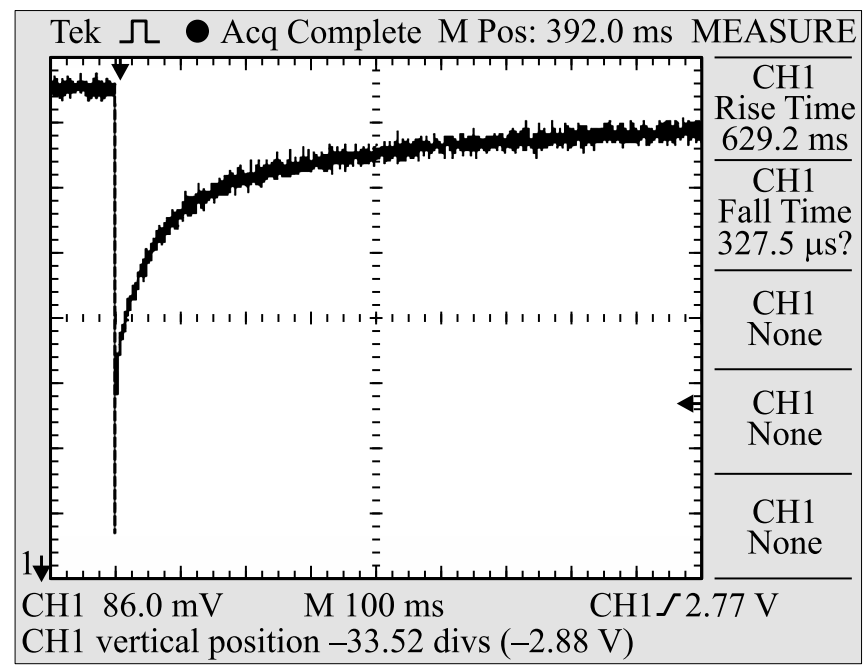

Pис. 1. Характерная осциллограмма прошедшего через кристалл BSO излучения после воздействия лазерного импульса с интенсивностью $5 \mathrm{MW} / \mathrm{cm}^{2}$.

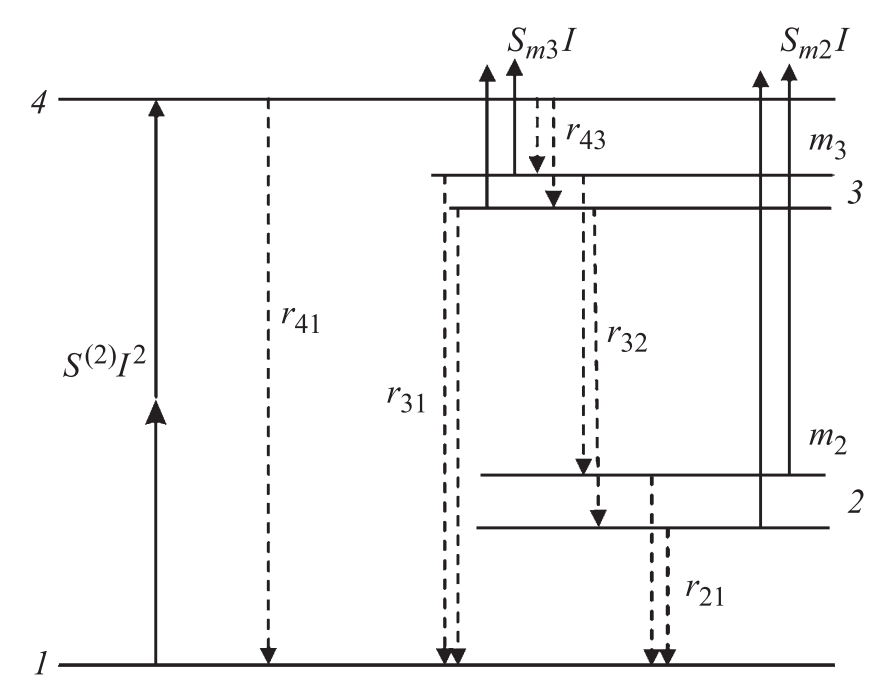

Рис. 2. Схема энергетических уровней и процессы фотовозбуждения, рекомбинации и захвата подвижных электронов в кристалле силиката висмута.

лазерных импульсов в рассмотрение включен механизм двухквантового поглощения света, скорость переходов при котором пропорциональна квадрату интенсивности падающего излучения.

Для описания фотоиндуцированного поглощения воспользуемся системой уравнений, полученной с учетом однофотонного поглощения электронами из ловушечных уровней и двухквантовыми переходами из валентной зоны в зону проводимости [19]:

$$
\begin{gathered}
\frac{\partial n}{\partial t}=\frac{\gamma}{2 \hbar} I_{0}^{2}+m_{3} S_{m 3} I+m_{2} S_{m 2} I \\
-r_{41} n p-r_{43} n\left(M_{3}-m_{3}\right), \\
\frac{\partial m_{3}}{\partial t}=-m_{3} S_{m 3} I+r_{43} n\left(M_{3}-m_{3}\right) \\
-r_{32} m_{3}\left(M_{32}-m_{2}\right)-r_{31} m_{3} p, \\
\frac{\partial m_{2}}{\partial t}=-m_{2} S_{m 2} I+r_{32} m_{3}\left(M_{2}-m_{2}\right)-r_{21} m_{2},
\end{gathered}
$$

где $n$ - концентрация электронов в зоне проводимости, $m_{2}$ и $m_{3}-$ концентрации электронов на долгоживущем и короткоживущем уровне соответственно, $M_{2}$ и $M_{3}-$ полные концентрации долгоживущих и короткоживущих ловушек, $p$ - концентрация дырок в валентной зоне, $\gamma$ - коэффициент двухквантового поглощения, $S_{m 2}$ и $S_{m 3}$ - сечения фотоионизации с соответствующих ловушечных уровней, $r_{i j}$ - коэффициенты рекомбинации.

Исходя из условия сохранения зарядов, количество дырок в валентной зоне равно суммарному количеству электронов в зоне проводимости и на ловушечных уровнях:

$$
p=n+m_{2}+m_{3} .
$$

Для удобства анализа процессов заселения и релаксации ловушечных уровней с учетом большого разброса 
скоростей переходов между различными состояниями с характерными временами жизни от микросекунд до десятков часов выделим несколько этапов.

Первый этап - возбуждение. Электроны из валентной зоны и ловушечных уровней при поглощении квантов света переходят в зону проводимости. На рис. 2 этому соответствуют переходы $1-4,2-4,3-4$. Система уравнений (1)-(3) преобразуется к виду

$$
\begin{gathered}
\frac{n}{\partial t}=\gamma 2 \hbar I_{0}^{2}+m_{3} S_{m 3} I_{0}+m_{2} S_{m 2} I_{0}, \\
\frac{m_{3}}{\partial t}=-m_{3} S_{m 3} I_{0}, \\
\frac{\partial m_{2}}{\partial t}=-m_{2} S_{m 2} I_{0} .
\end{gathered}
$$

Время жизни электронов в зоне проводимости крайне мало из-за большой скорости рекомбинации. В результате электроны возвращаются в валентную зону или переходят на короткоживущий уровень 3, лежащий вблизи зоны проводимости. Эти переходы можно считать вторым этапом (нано-, микросекундная релаксация) и описать уравнениями

$$
\begin{gathered}
\frac{\partial n}{\partial t}=-r_{41} n p-r_{43} n\left(M_{3}-m_{3}\right), \\
\frac{\partial m_{3}}{\partial t}=r_{43} n\left(M_{3}-m_{3}\right) .
\end{gathered}
$$

Третий этап, происходящий за миллисекунды, - релаксация, отвечающая переходу 3-1:

$$
\frac{\partial m_{3}}{\partial t}=-r_{31} m_{3} p
$$

При этом часть электронов захватывается долгоживущими ловушками (переход 3-2). Такой процесс имеет меньшую вероятность, но позволяет заселять долгоживущие уровни 2 с временем жизни несколько десятков часов при использовании большого количества лазерных импульсов [9].

При дальнейшем рассмотрении остановимся на уравнении (10), решение которого в приближении малой населенности долгоживущих ловушек $\left(m_{2}=0\right.$ и $\left.m_{3}=p\right)$ можно представить в виде

$$
m_{3}=\frac{m_{30}}{1+t / \tau}
$$

где $m_{30}-$ начальная концентрация электронов на ловушечном уровне, $\tau$ - характерное время жизни, которое обратно пропорционально коэффициенту рекомбинации $r_{31}$.

Однако данная зависимость испытывает существенные расхождения с полученными экспериментальными данными при малых временах жизни (менее $10 \mathrm{~ms}$ ). Учитывая большое количество разнообразных примесных и дефектных центров, характерных для кристаллов силиката висмута, для объяснения наблюдаемых расхождений предложено разбить ловушечный уровень 3

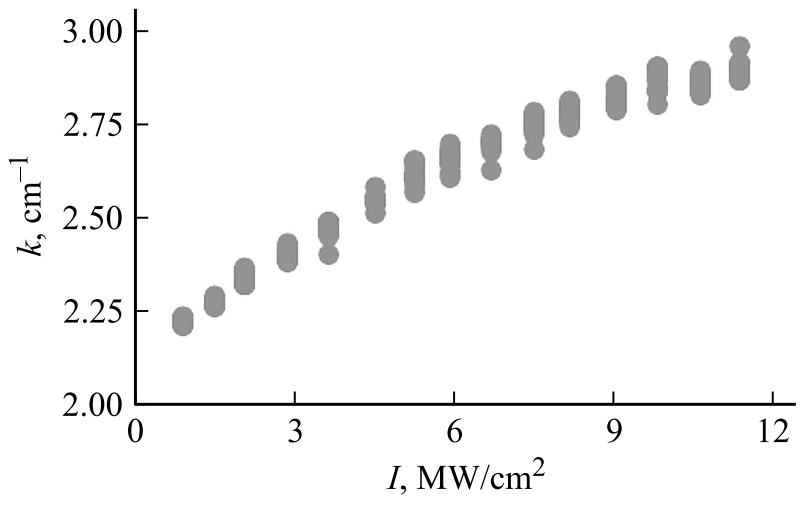

Рис. 3. Зависимость коэффициента поглощения от интенсивности импульсного излучения для кристалла BSO.

на два подуровня $3, a$ и $3, b$ с различными временами жизни. Для подуровня с меньшим временем жизни 3, $a$ решение уравнения (5) в условиях заметного заселения долгоживущего подуровня $3, b$ можно представить в виде

$$
m_{3 a}=m_{3 a 0} \exp \left(-t / \tau_{3 a}\right) .
$$

Для подуровня с большим временем жизни

$$
m_{3 b}=\frac{m_{3} b 0}{1+t / \tau_{b}} .
$$

С учетом уравнений (12), (13) коэффициент поглощения, связанный с заселением указанных подуровней, можно представить в виде

$$
k=k_{3 a 0} \exp \left(-\frac{t}{\tau_{3 a}}\right)+\frac{k_{3 b 0}}{1+t / \tau_{3 b}},
$$

где $k_{3 a 0}, k_{3 b 0}-$ начальные коэффициенты поглощения, связанные с соответствующими подуровнями, $\tau_{3 a}$ и $\tau_{3 b}$ - их времена жизни.

Как показал количественный анализ зарегистрированных осциллограмм, приведенное выражение удовлетворительно описывает экспериментальные результаты и может быть использовано для определения характерных времен релаксации.

На рис. 3 представлена зависимость коэффициента поглощения от интенсивности падающего излучения. Видно, что фотоиндуцированное поглощение эффективно проявляется при интенсивностях, превышающих $1 \mathrm{MW} / \mathrm{cm}^{2}$, причем при интенсивностях более $10 \mathrm{MW} / \mathrm{cm}^{2}$ происходит насыщение роста наведенного поглощения. Для нахождения характерных времен релаксации используем формулу (14). На рис. 4 представлены зависимости времени релаксации для ловушечных подуровней от интенсивности лазерного излучения. С увеличением интенсивности наблюдается уменьшение времени релаксации, которое выходит на постоянное значение при интенсивностях, превышающих $5 \mathrm{MW} / \mathrm{cm}^{2}$. При этом быстрые процессы релаксации характеризуются временами на уровне нескольких миллисекунд, а 


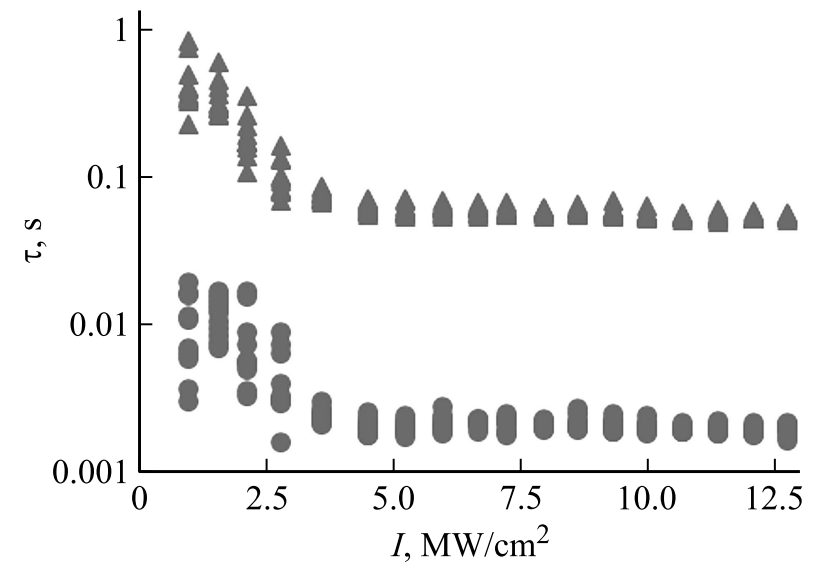

Рис. 4. Зависимость времени релаксации ловушечных подуровней от интенсивности падающего излучения.

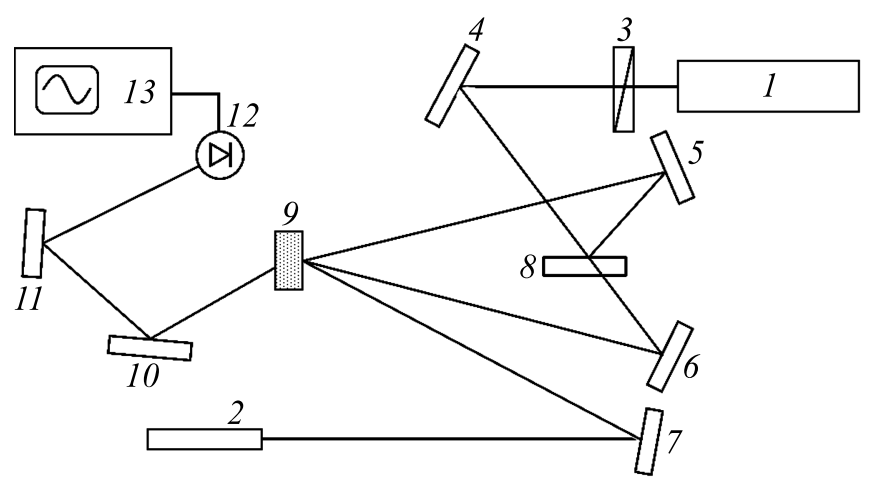

Рис. 5. Схема экспериментальной установки, где 1 - лазер на иттрий-алюминиевом гранате; 2 - гелий-неоновый лазер; 3 - полуволновая пластинка, 4-8, 10, 11 - зеркала; 9 кристалл силиката висмута; $12-$ скоростное фотоприемное устройство; 13 - цифровой осциллограф.

медленные процессы релаксируют с временами порядка $50 \mathrm{~ms}$.

\section{Импульсная запись динамических голограмм}

Проведенные исследования фотоиндуцированного поглощения в кристаллах силиката висмута позволили определить условия записи динамических голограмм при импульсном лазерном возбуждении. Для исследования динамики заселения и релаксации голографических решеток была использована экспериментальная установка, представленная на рис. 5.

В экспериментах использовалась вторая гармоника импульсного лазера на иттрий-алюминиевом гранате с неодимом с $\lambda=532 \mathrm{~nm}$ и длительностью импульса $20 \mathrm{~ns}$, маломощный непрерывный гелий-неоновый лазер и скоростное фотоприемное устройство с временным разрешением $20 \mu \mathrm{s}$, а также цифровой осциллограф с частотой $200 \mathrm{MHz}$. Система зеркал 10, 11 и светофильтры на входе фотодетектора 12 использовались для исключения вклада мощного импульсного излучения $\mathrm{Nd}$ : YAG-лазера в результаты эксперимента за счет пространственного и спектрального разделения дифрагированного пучка и импульсных пучков, формирующих голограмму.

Характерные осциллограммы дифрагированного излучения представлены на рис. 6. Детальный анализ осциллограмм на различных временных масштабах позволил выявить два механизма формирования динамических голограмм с временами релаксации, отличающимися на три порядка. При сравнительно небольших интенсивностях, порядка $1 \mathrm{MW} / \mathrm{cm}^{2}$, имеем дифрагированный сигнал с временем релаксации на уровне нескольких секунд. При интенсивностях, превышающих $5 \mathrm{MW} / \mathrm{cm}^{2}$, появляется быстрая компонента с временем релаксации на уровне миллисекунд. Вклад быстрого механизма записи динамических голограмм доминирует при больших интенсивностях лазерного излучения, превышающих $10-15 \mathrm{MW} / \mathrm{cm}^{2}$. Такую закономерность иллюстрирует рис. 7, на котором представлены зависимости времени формирования медленной компоненты решетки $(a)$ и отношения амплитуд медленной и быстрой компонент динамической голограммы $(b)$ от интенсивности записывающего голограмму излучения.

Количественный анализ динамики релаксации медленной и быстрой компонент динамической решетки показал, что удовлетворительная аппроксимация имеет место при использовании формулы, подобной (14) с разделением дифрагированного сигнала на две компоненты. На рис. 8 представлены зависимости времени релаксации быстрой и медленной компонент динамической решетки от интенсивности записывающего голограмму лазерного излучения. Видно, что времена релаксации медленной компоненты составляют секунды. В то же время быстрая компонента, которая появляется при интенсивностях более $5 \mathrm{MW} / \mathrm{cm}^{2}$, имеет характерные времена на уровне миллисекунд, причем при больших интенсивностях заметный вклад дает быстрая составляющая на уровне $100 \mu$ s.

\section{Заключение}

Таким образом, в работе проведены экспериментальные исследования динамики фотоиндуцированного поглощения и записи динамических голограмм в фоторефрактивных кристаллах силиката висмута мощными лазерными импульсами длительностью $20 \mathrm{~ns}$ на длине волны $532 \mathrm{~nm}$. Показано, что наведенное поглощение заметно проявляется при интенсивностях, превышающих $1 \mathrm{MW} / \mathrm{cm}^{2}$, при этом заселяются короткоживущие ловушечные уровни с временами релаксации на уровне единиц и десятков миллисекунд. В этих условиях реализована запись динамических голограмм и показана возможность формирования голографических решеток 

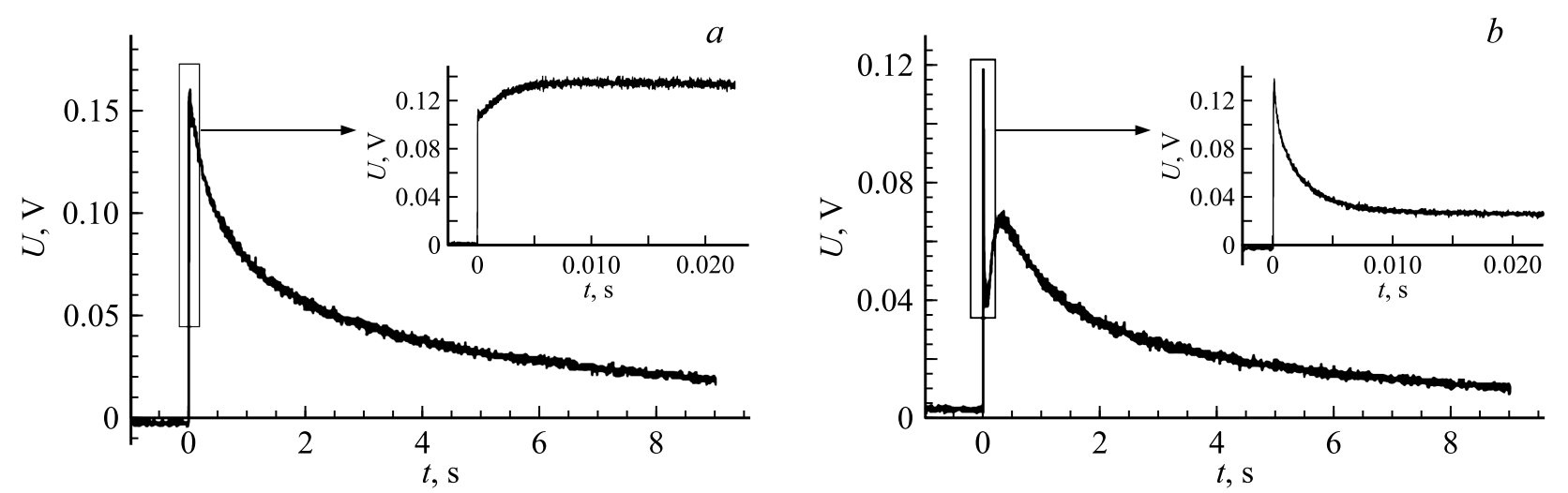

Рис. 6. Осциллограммы дифрагированного сигнала при интенсивности лазерного излучения $3 \mathrm{MW} / \mathrm{cm}^{2}(a)$ и $15 \mathrm{MW} / \mathrm{cm}^{2}(b)$.
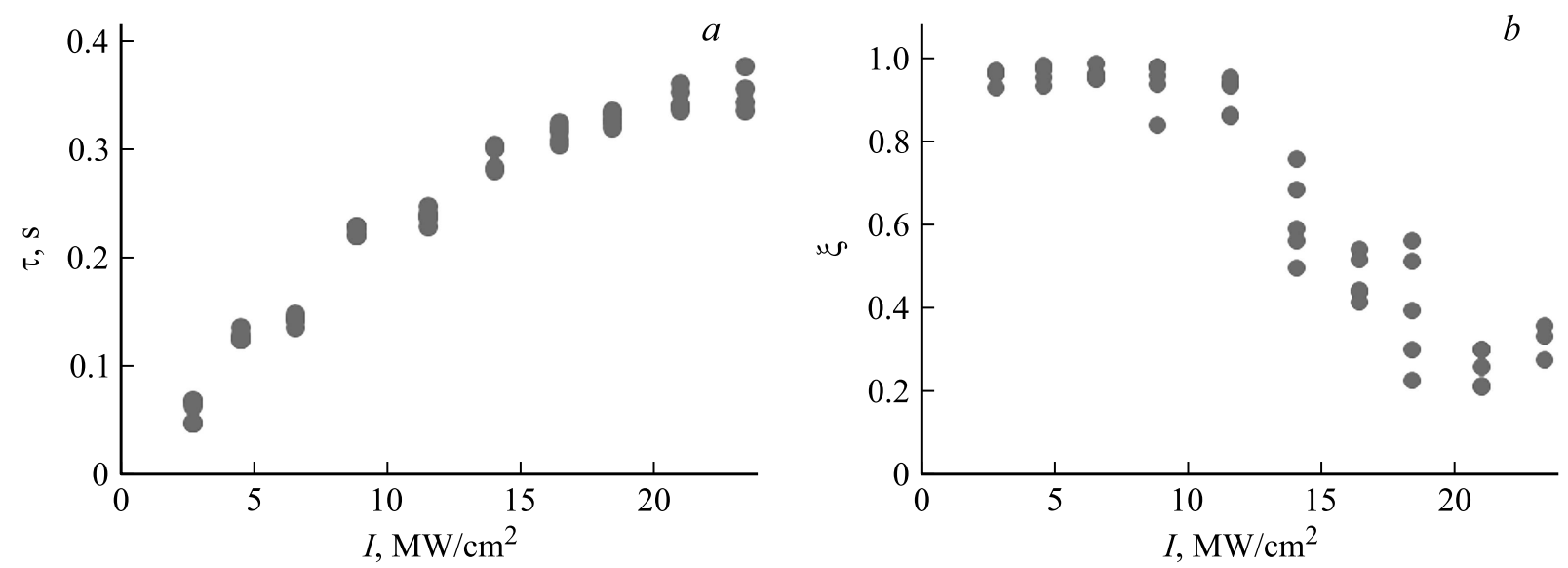

Рис. 7. Зависимости времени формирования долгоживущей решетки $(a)$ и коэффициента, характеризующего отношение амплитуд медленной и быстрой компонент динамической голограммы $(b)$, от интенсивности лазерного излучения.
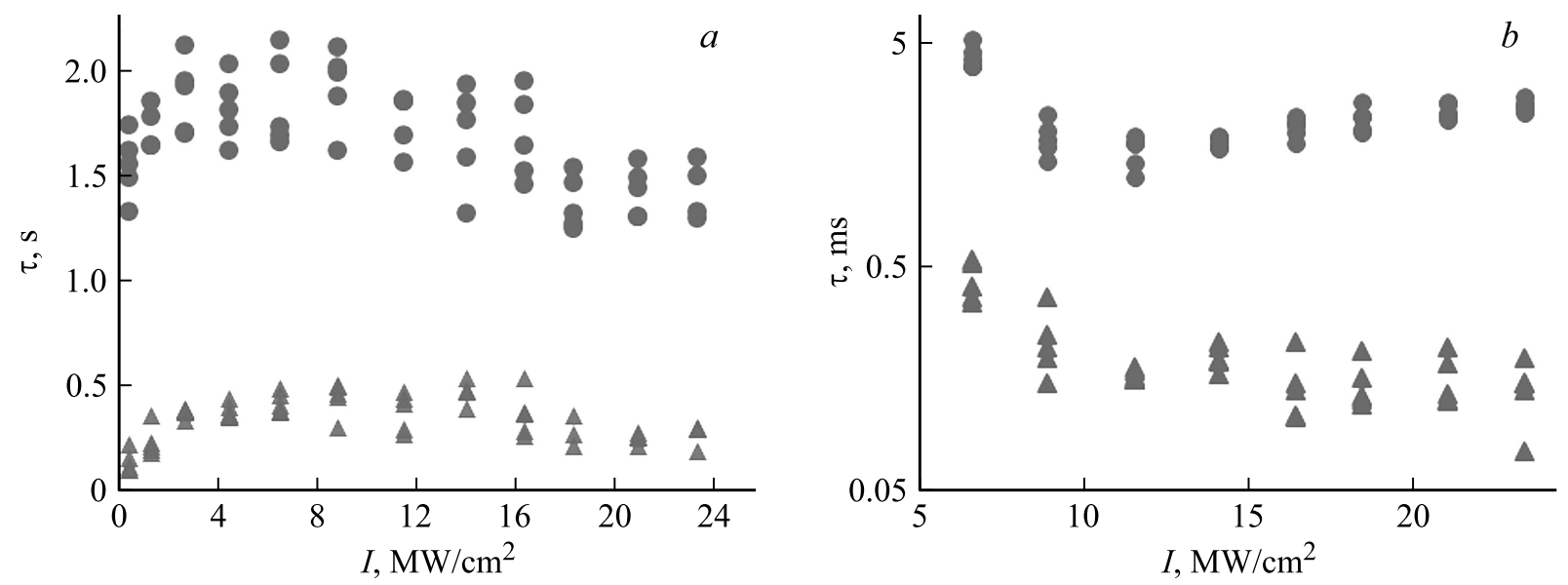

Рис. 8. Зависимость времени релаксации быстрой и медленной компонент динамической решетки от интенсивности записывающего голограмму лазерного излучения.

как с временами на уровне миллисекунд, так и на уровне секунд. Сравнение динамики релаксации фотоиндуцированного поглощения и голографических решеток позволило сделать вывод, что быстрый механизм формирования динамических решеток можно связать с заселением короткоживущих ловушечных уровней с миллисекундными временами релаксации. Второй механизм записи решеток с временами релаксации на уровне секунд связан с формированием фоторефрактивной нелинейности в поле пространственного заряда. Установлено, что при 
больших интенсивностях преимущественно заселяются короткоживущие ловушки, препятствующие диффузии электронов в зоне проводимости и формированию пространственного заряда, связанного с долгоживущими ловушечными уровнями.

\section{Список литературы}

[1] Петров М.П., Степанов С.И., Хоменко А.В. Фоторефрактивные кристаллы в когерентной оптике. СПб.: Наука, 1992.

[2] Jones D.C., Lyuksyutov S.F., Solymar L. // Appl. Phys. B. 1991. V. 52. P. 173?175. doi 10.1007/bf00750947

[3] Buse K. // Appl. Phys. B: Lasers a. Optics. 1997. V. 64. N 3. P. 273-291. doi 10.1007/s003400050175

[4] Nazhestkina N.I., Kamsyilin A.A., Kobozev O.V., Prokofiev V.V. // Appl. Phys. B. 2001. V. 72. N 6. P. 767-773.

[5] Каргин Ю.Ф., Бурков В.И., Марьин А.А., Егорышев А.В. Кристаллы $\mathrm{Bi}_{12} \mathrm{Si}_{x} \mathrm{O}_{20-\delta}$ со структурой силленита. Синтез, строение, свойства. М.: Изд-во ИОХН РАН, 2004.

[6] Шандаров С.М., Буримов Н.И., Кульчин Ю.Н., Ромашко Р.В., Толстик А.Л., Шепелевич В.В. // Квант. электрон. 2008. T. 38. № 11. C. 1059-1069; Shandarov S.M., Burimov N.I., Kul'chin Yu.N., Romashko R.V., Tolstik A.L., Shepelevich V.V. // Quantum Electronics. 2008. V. 38. N 11. P. 1059-1069. doi 10.1070/qe2008v038n1 labeh013793

[7] Pochi Yeh. Introduction to Photorefractive Nonlinear Optics. NY: Wiley, 1993.

[8] Wevering S., Imbrock J., Kratzig E. // J. Opt. Soc. Am. B. 2001. V. 18. P. 472-478. doi 10.1364/josab.18.000472

[9] Matusevich A., Tolstik A., Kisteneva M., Shandarov S., Matusevich V., Kiessling A., Kowarschik R. // Appl. Phys. B. 2008. V. 92. N 2. P. 219-224. doi 10.1007/s00340-008-3098-z

[10] Matusevich A., Tolstik A., Kisteneva M., Shandarov S., Matusevich V., Kiessling A., Kowarschik R. // Appl. Phys. B. 2009. V. 96. N 1. P. 119-125. doi 10.1007/s00340-009-3512-1

[11] Кистенева М.Г., Акрестина А.С., Сивун Д.О., Киселев Р.В., Шандаров С.М., Смирнов С.В., Толстик А.Л., Агишев И.Н., Станкевич А.В., Каргин Ю.Ф. // Докл. Томского государственного университета систем управления и радиоэлектроники. 2010. Т. 22. № 2. Ч. 2. С. 62-65.

[12] Khudyakova E.S., Kisteneva M.G., Shandarov S.M., Kornienko T.A., Tolstik A.L., Kargin Yu.F. // Radiophysics and Quantum Electronics. 2015. V. 57. N 8-9. C.589-594. doi 10.1007/s11141-015-9543-z

[13] Kornienko T., Kisteneva M., Shandarov S., Tolstik A. // Phys. Proc. 2017. V. 86. P. 105-112. doi j.phpro.2017.01.029

[14] Толстик А.Л., Матусевич А.Ю., Кистенева М.Г., Шандаров С.М., Иткин С.И., Мандель А.Е., Каргин Ю.Ф., Кульчин Ю.Н., Ромашко Р.В. // Квант. электрон. 2007. T. 37. № 11. C. 1027-1032; Tolstik A.L., Matusevich A.Yu., Kisteneva M.G., Shandarov S.M., Itkin S.I., Mandel' A.E., Kargin Yu.F., Kul'chin Yu.N., Romashko R.V.// Quantum Electronics. 2007. V. 37. N 11. P. 1027-1032. doi 10.1070/qe2007v037n11abeh013371

[15] Hermann J.P., Herriau J.P., Huignard J.P. // Appl. Opt. 1981. V. 20. P. 2173-2174. doi 10.1364/AO.20.002173

[16] Partanen J.P., Nouchi P., Jonathan J.M.C., Hellwarth R.W. // Phys. Rev. B. 1991. V. 44. P. 1487-1491.

doi 10.1103/physrevb.44.1487
[17] Murillo J.G. // Optics Commun. 1999. V. 159. P. 293-300. doi 10.1016/s0030-4018(98)00610-5

[18] Станкевич А.В., Толстик А.Л., Хайдер Х.К. // Письма в ЖТФ. 2011. Т. 37. № 16. С. 7-14; Stankevich A.V., Tolstik A.L., Haider H.K. // Technical Physics Letters. 2011. V. 37. P. 746-749. doi 10.1134/s1063785011080268

[19] Толстик А.Л. Ханон Х.К. // Вестник Белорусского государственного университета. Сер 1. Физика. Математика. Информатика. 2012. № 2. С. 3 ?7. 